\title{
ANÁLISE DOS AERODISPERSOIDES SÓLIDOS PRODUZIDOS NA INDUSTRIALIZAÇÃO DA MADEIRA
}

\author{
Elenise Leocádia da Silveira Nunes ${ }^{1}$, João Carlos Moreschi ${ }^{2}$ \\ ${ }^{1}$ Desenho Industrial, Dra ${ }^{\mathrm{a}}$., Depto. Desenho Industrial, UTFPR, Curitiba, PR, Brasil - elenise@utfpr.edu.br \\ ${ }^{2}$ Eng. Florestal, Dr., Depto. de Engenharia e Tecnologia Florestal, UFPR, Curitiba, PR, Brasil - moreschi@ membracel.com.br
}

Recebido para publicação: 01/12/2008 - Aceito para publicação: 14/04/2009

\begin{abstract}
Resumo
Este estudo buscou caracterizar o particulado sólido proveniente de painéis de madeira aglomerada, visando minimizar a exposição ocupacional sofrida pelos trabalhadores do setor de base florestal. Para tanto, foram utilizados métodos de análise regulamentados por órgãos normatizadores, como a American Conference of Governmental Industrial Hygienists (ACGIH), a National Institute for Occupational Safety and Health (NIOSH), a Occupational Safety and Health Administration (OSHA), a American Society for Testing and Materials (ASTM) e a Fundação Jorge Duprat Figueiredo de Segurança e Medicina do Trabalho (FUNDACENTRO). Os resultados demonstraram que o particulado sólido do aglomerado é um material potencialmente tóxico e passível de desenvolver doenças ocupacionais nos trabalhadores expostos por longo período de tempo.

Palavras-chave: Aerodispersoide sólido; poeira sólida do aglomerado; risco ocupacional.
\end{abstract}

\begin{abstract}
Solid airborne analysis produced in wood industrialization. The aim of this study was to feature the solid airborne dust generated from the mechanic processing of agglomerated wood particleboard in order to minimize the risk of occupational exposing that the workers could be subjected to. As analyzing tools there were used solid airborne analyzing rules and methods regulated by world known regulatory agencies: the American Conference of Governmental Industrial Hygienists (ACGIH), the National Institute for Occupational Safety and Health (NIOSH), the Occupational Safety and Health Administration (OSHA), the American Society for Testing and Materials (ASTM) and Fundação Jorge Duprat Figueiredo de Segurança e Medicina do Trabalho (FUNDACENTRO). This way the solid particulate from the agglomerated had been characterized as potentially toxic and it could induce occupational diseases in the workers exposed to it for a long period of time.

Keywords: Solid airborne; agglomerated solid dust; occupational risk.
\end{abstract}

\section{INTRODUÇÃO}

O setor de transformação da indústria de base florestal utiliza em grande escala painéis de madeira aglomerada como matéria-prima para a produção de bens de consumo, como peças de mobiliário e embalagens. Entretanto, o processamento mecânico (corte, usinagem e lixamento) de painéis de madeira aglomerada tem como resultado final, além do produto acabado, a produção de uma grande quantidade de poeira, em diversas granulometrias, que fica em suspensão no ambiente de trabalho. Com o objetivo de reduzir a concentração desse tipo de resíduo, as empresas de médio e grande porte utilizam sistemas de exaustão na fonte, ou seja, aspiração da poeira gerada no instante em que ocorre o processamento do painel. Entretanto, empresas de pequeno porte ou com recursos tecnológicos mais escassos, acumulam esse material residual em um local determinado dentro do layout da empresa e só efetuam a limpeza e o descarte desse material uma vez por semana ou a cada quinze dias.

Segundo a American Conference of Governmental Industrial Hygienists (ACGIH, 1991), a exposição ao pó de madeira pode causar problemas à saúde dos trabalhadores. Essa exposição pode ser classificada de duas formas: a exposição direta, quando o trabalhador está sujeito ao contato direto com a poeira gerada, pois efetua o processamento da madeira, e a exposição indireta, quando o pó de madeira fica disperso no ambiente e todos os trabalhadores entrarão em contato com ele através do ar 
contaminado. A exposição indireta ao pó de madeira pode causar irritação nos olhos e mucosas, dermatites por contato e alérgicas, eritemas e problemas respiratórios, como alergias, sinusites, asma e bronquite. Por outro lado, e de forma mais grave, a exposição direta ao pó de madeira pode transformar esses problemas em doenças crônicas, inclusive com o aparecimento de carcinogêneres.

Sendo assim, o objetivo deste estudo foi caracterizar o aerodispersoide sólido produzido a partir do processamento mecânico de painéis de madeira aglomerada, através da determinação do seu limite de tolerância e concentração de partículas, buscando minimizar o risco de doenças ocupacionais para os trabalhadores expostos.

\section{REVISÃO BIBLIOGRÁFICA}

\section{Características do painel de madeira aglomerada}

Segundo Iwakiri (2005), painel de madeira aglomerada, comercialmente denominado de "aglomerado", é um painel produzido com partículas de madeira tipo sliver, reconstituído a partir de uma matriz randômica e consolidado através da aplicação de calor e pressão, com a incorporação de um adesivo sintético. É importante ressaltar que a qualidade técnica dos painéis de madeira aglomerada é descrita pelas suas propriedades físicas, que devem estar em consonância com a norma EN 312 (2003). A característica principal do painel aglomerado é possuir comportamento elástico-mecânico igual ou maior que a madeira maciça, sendo que propriedades como flexão estática e ligação interna só são afetadas com a variação dos elementos dimensionais das partículas. É importante ressaltar que a maior parte do aglomerado produzido no Brasil é elaborada a partir de partículas de Pinus spp. provenientes de florestas plantadas, o que faz com que a qualidade do painel seja relativamente maior do que em outros países. Resíduos provenientes de serrarias e de outros setores do processamento mecânico da madeira são utilizados na geração de partículas, entretanto em pequenas proporções e apenas por alguns fabricantes.

Normatizada pela American Society for Testing and Materials - ASTM D4690-99 (2005), a resina UF é a mais utilizada na fabricação de painéis de madeira aglomerada para uso interno. Sua denominação é polímero termofixo de ureia-formaldeído em solução aquosa. É um adesivo de fácil manuseio, solúvel em água, cura rápida e incolor que não agrega alto custo ao produto final. Sua proporção de utilização é em torno de 6 a 12\% da massa seca de partículas e pode atuar em diferentes temperaturas de prensagem, de 90 a $120{ }^{\circ} \mathrm{C}$, dependendo do tipo do painel a ser fabricado e da prensa utilizada.

\section{Aerodispersoide sólido}

De acordo com a Fundação Jorge Duprat Figueiredo de Segurança e Medicina do Trabalho (FUNDACENTRO), aerodispersoide sólido é a partícula formada a partir da ruptura mecânica de um material sólido seco, seja por corte, quebra, usinagem, fricção ou fundição, capaz de se manter suspensa no ar por tempo suficiente para que, com equipamentos específicos, possa ser efetuada a sua observação e medição. O tempo de suspensão depende diretamente do tamanho da partícula, de seu peso específico e da velocidade de movimentação do ar no ambiente analisado (NHO 03, 2001).

Com o intuito de regulamentar o limite de exposição ao pó de madeira, em 1985 foi criado o Occupational Safety and Health Review Commission (OSHC), uma comissão composta por diversas organizações normatizadoras internacionais, que, a partir de estudos específicos, criaram valores de limite de tolerância para esse tipo de aerodispersoide. Dessa forma, o valor do limite de tolerância (TLV Threshold Limit Value) determinado pela ACGIH (2006) ao pó de madeira maciça é de $1 \mathrm{mg} / \mathrm{m}^{3} \mathrm{para}$ madeiras duras e $5 \mathrm{mg} / \mathrm{m}^{3}$ para madeiras moles ou gimnospermas, para um período de 8 horas diárias ou 40 horas semanais. Entretanto, o limite máximo de exposição determinado para madeiras moles é de 10 $\mathrm{mg} / \mathrm{m}^{3}$, por períodos de 15 minutos com intervalos de 60 minutos entre exposições consecutivas. Para o National Institute for Occupational Safety and Health (NIOSH, 1992), o limite de exposição recomendado (REL - Recommended Exposure Limit) ao pó de madeira é de $1 \mathrm{mg} / \mathrm{m}^{3}$, tanto para madeiras moles quanto madeiras duras, para uma jornada de trabalho de até 10 horas diárias e/ou 40 horas semanais. Para o Occupational Safety and Health Administration (OSHA, 1994), o limite de exposição recomendado (PEL - Permissible Exposure Limit) é de $15 \mathrm{mg} / \mathrm{m}^{3}$ para poeira total e $5 \mathrm{mg} / \mathrm{m}^{3}$ para a fração de poeira respirável, independentemente de madeira mole ou madeira dura. É importante ressaltar 
que cientificamente as madeiras duras são denominadas de angiospermas e as madeiras moles de gimnospermas (BURGER; RICHTER, 1991).

Por outro lado, quanto à poeira proveniente do processamento de painéis de madeira reconstituída, formados a partir de partículas de madeira unidas através de um adesivo termofixo, sob efeito de calor e pressão, não se tem limite de tolerância determinado por órgãos regulamentadores. Existe apenas a indicação na tabela da ACGIH (1998) de que esse tipo de particulado se enquadra na categoria de Particulates not Otherwise Regulated (PNOC) - Particulado Não Classificado de Outra Maneira - ou seja, é um particulado potencialmente tóxico, com percentual de sílica livre cristalina menor que $1 \%$, mas sem limite de tolerância e limite de concentração definidos.

De acordo com a ACGIH (1998), existe uma relação entre o tamanho do aerodispersoide sólido e o local de penetração deles no sistema respiratório. Nesse sentido, têm-se:

- Particulado inalável: diâmetro médio entre 25 e $100 \mu \mathrm{m}$. Devido ao seu tamanho passa pelas fossas nasais e pela boca, sendo eliminado pelo sistema de filtragem do sistema respiratório.

- Particulado torácico: diâmetro médio entre 10 e $25 \mu \mathrm{m}$. Atinge a faringe, a traqueia e os brônquios, mas ainda é passível de ser eliminado pelo sistema de filtragem do organismo humano.

- Particulado respirável: diâmetro médio entre 1 e $10 \mu \mathrm{m}$. Por possuir tamanho reduzido, passa pelos demais órgãos de filtragem do sistema respiratório e se deposita na região alveolar.

É importante ressaltar que o sistema respiratório humano é constituído por vários órgãos que conduzem o ar inalado para dentro e para fora das cavidades pulmonares, e por meio de mecanismos de filtragem promovem a proteção do organismo contra agentes externos. Assim sendo, partículas maiores são capturadas pelas fossas nasais e vias aéreas superiores e são eliminadas através da limpeza mucociliar pela tosse. Já as partículas solúveis se depositam sobre qualquer tecido do aparelho respiratório e produzem irritação até a sua dissolução completa. Partículas pequenas se depositam profundamente na cavidade pulmonar e, dependendo do seu nível de toxicidade, podem ser dissolvidas e absorvidas pelo sangue ou podem ser sedimentadas pelas células macrófagas.

De forma mais abrangente para a ACGIH (1998), a toxicidade do aerodispersoide sólido também depende das condições ambientais do local de trabalho e das condições físicas do trabalhador. As condições ambientais são: concentração de partículas, temperatura ambiente, umidade relativa do ar e velocidade do ar. As condições físicas do trabalhador são: a variabilidade individual, a saúde, o sexo, a idade e a forma de realização das tarefas. É importante ressaltar que a maioria dos trabalhadores, quando possui algum tipo de obstrução nas fossas nasais, seja por enfermidade ou por irritação alérgica, respira pela boca, e dessa maneira o ar contaminado é direcionado diretamente aos pulmões, sem passar pelo sistema de filtragem das vias aéreas superiores. Assim sendo, para a avaliação do risco à saúde do trabalhador, o particulado sólido deve ser analisado principalmente nas frações de poeira inalável e respirável, sendo que, nesta última, o equipamento utilizado para efetuar a coleta das partículas deve simular a respiração humana (ACGIH, 1998).

\section{MATERIAIS E MÉTODOS}

Visando minimizar as influências das variáveis ambientais, principalmente de particulados sólidos proveniente de outros tipos de madeira ou materiais diversos, a presente pesquisa foi realizada através de um estudo de caso em uma indústria produtora de painéis de madeira aglomerada, e a coleta de amostras ocorreu em um setor específico, que refila os painéis aglomerado em medidas especiais.

Os tipos de amostragem mais utilizados para determinação da concentração de particulado sólido em análises de exposição ocupacional são amostragem pessoal ou poeira respirável (PR) e amostragem total ou poeira total (PT). Na fração de poeira respirável (PR), o aparelho utilizado para coleta do particulado sólido é colocado próximo à zona de respiração do trabalhador, enquanto este realiza a sua atividade diária, simulando o sistema de filtragem do aparelho respiratório, pois separa apenas as partículas respiráveis, permitindo avaliar a exposição ocupacional direta dos trabalhadores. Na fração de poeira total (PT), o aparelho para coleta do particulado é colocado em um local predeterminado no layout fabril, com grande circulação de trabalhadores e próximo às fontes geradoras de poeira, coletando qualquer tamanho de partícula dispersa no ar do ambiente de trabalho, o que permite a avaliação da exposição ocupacional indireta dos trabalhadores envolvidos no processo. Assim sendo, de acordo com a 
ACGIH (1998), o valor do limite de tolerância (TLV - Threshold Limit Value) para o Particulado Sólido não Classificado de Outra Maneira (PNOC) é de $8,8 \mathrm{mg} / \mathrm{m}^{3}$ para a fração de poeira total (PT) e 2,64 $\mathrm{mg} / \mathrm{m}^{3}$ para a fração de poeira respirável (PR).

Assim, foram coletadas amostras do particulado sólido nas frações de poeira total (PT) e poeira respirável (PR) durante a atividade de trabalho, por meio de equipamentos de amostragem específicos para cada coleta. O material particulado para análise de poeira respirável (PR) foi coletado com bomba gravimétrica autoajustável da marca MSA, acoplada a um ciclone separador de partículas de nylon, sobre filtro de membrana de PVC de $37 \mathrm{~mm}$. O material particulado para análise de poeira total (PT) foi coletado de duas formas: com a utilização da bomba gravimétrica autoajustável da marca MSA sobre o filtro de membrana de PVC de $37 \mathrm{~mm}$ e em frascos de polipropileno, nos quais foi coletado o particulado sólido depositado sobre o maquinário, uma hora após o término da atividade de trabalho, ou seja, quando a maior parte do particulado já havia se depositado sobre a bancada de trabalho sob o efeito da gravidade.

As técnicas de análise utilizadas para o particulado sólido do aglomerado coletado foram gravimetria, microscopia ótica, microscopia eletrônica de varredura, espectrofotometria de energia dispersiva (EDS), espectrofotometria na região do infravermelho e cromatografia líquida de alto desempenho, segundo os seguinte métodos:

- NHO 03 / FUNDACENTRO: Análise Gravimétrica de Aerodispersoides Sólidos Coletados Sobre Filtros de Membrana. Norma de Higiene Ocupacional, 2001.

- PV 2121 / OSHA: Gravimetric Determination Partially Validated, 2003.

- ASTM E112-96: Standard Test Methods for Determining Average Grain Size (Reapproved 2004).

- ASTM E1382-97:Standard Test Methods for Determining Average Grain Size Using Semiautomatic and Automatic Image Analysis (Reapproved 2004).

- Method 7602 / NIOSH - SILICA, CRYSTALLINE BY IR (K BR PELLET), in Manual of Analytical Methods (NMAM, 2003).

- Method 2016 / NIOSH - FORMALDEHYDE, in Manual of Analytical Methods (NMAM, 2003).

- Method 0500 / NIOSH - PARTICULATES NOT OTHERWISE REGULATED, TOTAL, Issue 2, de $15 / 08 / 1994$.

- Method 0600 / NIOSH - PARTICULATES NOT OTHERWISE REGULATED, RESPIRABLE, Issue 3, de 15/01/1998.

\section{RESULTADOS E DISCUSSÕES}

A determinação do risco ocupacional gerado pelo contato com o particulado sólido do aglomerado suspenso no ar do ambiente de trabalho foi realizada a partir da análise estatística dos dados obtidos, assim como através da comparação desses valores com os limites de tolerância estabelecidos por órgãos normatizadores, como a ACGIH, a NIOSH e a OSHA.

O resíduo disperso no ar caracteriza a concentração de partículas pertinente ao ambiente de trabalho analisado, sendo dependente do tipo de processamento efetuado, do material processado e das condições ambientais existentes. Na presente pesquisa, foi analisado o particulado sólido obtido a partir do corte retilíneo de painéis de madeira aglomerada com espessura média de $15 \mathrm{~mm}$, efetuado por uma serra seccionadora. A tabela 1 apresenta a síntese dos valores obtidos para o limite de tolerância (LT) e para a concentração de partículas $(\mathrm{C})$, relativos à fração de poeira total $(\mathrm{PT})$ e à fração de poeira respirável (PR).

De acordo com os dados apresentados na tabela 1, existe risco ocupacional para o trabalhador exposto ao particulado sólido do aglomerado na fração de poeira total (PT), pois a concentração de partículas encontrada no material amostrado $\left(\mathrm{C}_{\mathrm{pPT}}=16,069 \mathrm{mg} / \mathrm{m}^{3}\right)$ foi superior tanto ao limite de tolerância calculado especificamente para o particulado sólido do aglomerado $\left(\mathrm{LT}_{\mathrm{pPT}}=6,9994 \mathrm{mg} / \mathrm{m}^{3}\right)$ como para o limite de tolerância estabelecido pela ACGIH $\left(\operatorname{LT}_{\text {ACGIH }}=8,8 \mathrm{mg} / \mathrm{m}^{3}\right)$.

Com relação aos valores obtidos para a fração de poeira respirável (PR), como foi realizada a análise em apenas uma amostra, não foi possível efetuar uma análise efetiva dos dados no cálculo do limite de tolerância específico para o particulado sólido do aglomerado $\left(\mathrm{LT}_{\mathrm{pPR}}\right)$. Mas se for comparada a concentração de partículas encontrada para a fração respirável $\left(\mathrm{C}_{\mathrm{pPR}}=2,4741 \mathrm{mg} / \mathrm{m}^{3}\right) \mathrm{com}$ o limite de tolerância estabelecido pela $\mathrm{ACGIH}\left(\mathrm{LT}_{\mathrm{ACGIH}}=2,64 \mathrm{mg} / \mathrm{m}^{3}\right)$, tem-se valores muito próximos, o que 
indica potencial risco ocupacional para o trabalhador exposto ao particulado do aglomerado na fração respirável.

Tabela 1. Síntese dos valores do limite de tolerância (LT) e da concentração de partículas (C) obtidos no ambiente de trabalho analisado na presente pesquisa.

Table 1. Value synthesis of tolerance limit (LT) and particle concentration (C) obtained in work environment under analysis in this research.

\begin{tabular}{|c|c|c|c|}
\hline Especificação das informações levantadas & $\begin{array}{c}\text { Valor médio } \\
\text { obtido }\left(\mathrm{mg} / \mathrm{m}^{3}\right)\end{array}$ & $\begin{array}{c}\text { Desvio padrão } \\
\text { dp }\left(\mathbf{m g} / \mathbf{m}^{3}\right)\end{array}$ & $\begin{array}{c}\text { Parâmetro de } \\
\text { comparação }\left(\mathrm{mg} / \mathrm{m}^{3}\right)\end{array}$ \\
\hline $\begin{array}{l}\text { Limite de tolerância específico para o particulado } \\
\text { sólido do aglomerado }=\mathrm{LT}_{\mathrm{PT}} \text { (poeira total/PT). }\end{array}$ & $\mathrm{LT}_{\mathrm{PT}}=6,9994$ & 0,3073 & $C_{\mathrm{PT}}=13,6553$ \\
\hline $\begin{array}{l}\text { Limite de tolerância específico para o particulado } \\
\text { sólido do aglomerado }=\mathrm{LT}_{\mathrm{PT}} \text { (material coletado } \\
\text { sobre o maquinário). }\end{array}$ & $\mathrm{LT}_{\mathrm{PT}}=6,9468$ & 0,2963 & $C_{\mathrm{PT}}=16,1069$ \\
\hline $\begin{array}{l}\text { Concentração de partículas coletadas }=\mathrm{C}_{\mathrm{PT}} \text { (poeira } \\
\text { total/PT). }\end{array}$ & $C_{\mathrm{PT}}=16,1069$ & 2,2966 & $\begin{array}{l}\mathrm{LT}_{(\mathrm{ACGIH})}=8,8 \\
\mathrm{LT}_{\mathrm{PT}}=6,9994\end{array}$ \\
\hline $\begin{array}{l}\text { Concentração de partículas de poeira respirável = } \\
\mathrm{C}_{\mathrm{PR}} \text { (poeira respirável/PR). }\end{array}$ & $C_{P R}=2,4741$ & 0,3832 & $\begin{array}{c}\mathrm{LT}_{(\mathrm{ACGIH})}=2,64 \\
\mathrm{LT}_{\mathrm{PR}}=3,6364\end{array}$ \\
\hline $\begin{array}{l}\text { Limite de tolerância específico para o particulado } \\
\text { sólido do aglomerado }=\mathrm{LT}_{\mathrm{PR}} \text { (poeira respirável/PR) }\end{array}$ & $\mathrm{LT}_{\mathrm{PR}}=3,6364$ & $* * *$ & $C_{P R}=2,4741$ \\
\hline
\end{tabular}

LT: limite de tolerância; C: concentração de partículas; PT: fração de poeira total; PR: fração de poeira respirável; ***: limite de tolerância definido a partir de uma amostra, portanto sem variação estatística; LT $_{\mathrm{ACGIH}}$ : Limite de tolerância da ACGIH para "Particulado Não Classificado de Outra Maneira" (PNOC). Segundo a ACGIH, se C >LT existe risco ocupacional.

A toxidade do particulado sólido do aglomerado foi avaliada a partir da concentração de sílica cristalina e de formaldeído, sendo que na análise efetuada por EDS (Espectrômetro de Energia Dispersiva) foram detectadas partículas contendo uma concentração de silício variando de $11,74 \%$ a 39,74\%. De acordo com o OSHA (2002), na análise da concentração de sílica cristalina $\left(\mathrm{C}_{\mathrm{s}}\right)$ em aerodispersoides sólidos, a maior importância está na caracterização das partículas na fração respirável, pois quando o ar inalado chega aos pulmões com esse tipo de substância, ele pode promover a redução da capacidade respiratória e da elasticidade pulmonar, culminando por desenvolver silicose. Entretanto é importante ressaltar que a gravidade da silicose depende também do tempo de exposição ao agente agressor a que o trabalhador esteve sujeito durante a atividade de trabalho. O limite de tolerância da ACGIH (TLV - Threshold Limit Value) para a sílica livre cristalina é de $0,022 \mathrm{mg} / \mathrm{m}^{3}$, e para o formaldeído é de $0,369 \mathrm{mg} / \mathrm{m}^{3}$.

Segundo os resultados demonstrados na tabela 2, o particulado sólido do aglomerado apresentou na fração de poeira total (PT) concentração de sílica livre cristalina $\left(\mathrm{C}_{\mathrm{S}}\right) \quad 170 \%$ superior ao limite estabelecido pela ACGIH $\left(\mathrm{LT}_{\mathrm{ACGIH}}\right)$, o que indica grande risco ocupacional para os trabalhadores expostos. Entretanto, como esse tipo de particulado possui dimensões maiores que $10 \mu \mathrm{m}$, ou seja, pertence à fração inalável, pode ser eliminado pelo sistema de defesa do organismo humano através das vias respiratórias superiores. $\mathrm{Na}$ análise da fração de poeira respirável (PR) do particulado sólido do aglomerado, a concentração de sílica cristalina $\left(\mathrm{C}_{\mathrm{s}}\right)$ ficou $80 \%$ abaixo do limite de exposição estabelecido pela ACGIH $\left(\mathrm{LT}_{\mathrm{ACGIH}}\right)$. Esses dados indicam que grande parte do particulado pode ser filtrado pelo sistema respiratório superior do ser humano e provavelmente não chegará a penetrar nos pulmões.

Com relação ao formaldeído, foi detectada no particulado sólido do aglomerado uma concentração de formaldeído $\left(\mathrm{C}_{\mathrm{f}}\right) 60 \%$ superior aos limites de tolerância estabelecidos pela ACGIH $\left(\mathrm{LT}_{\mathrm{ACGIH}}\right)$. De acordo com a ACGIH, se a concentração de formaldeído encontrada no material amostrado for superior ao limite estabelecido, o material é caracterizado como substancia A2, ou seja, como substância suspeita de desenvolver carcinogêneres nos seres humanos. Entretanto, a toxidade do formaldeído tende a ser mais potencializada no instante em que o particulado sólido desprende do painel de madeira aglomerada durante o processamento mecânico do material (corte, usinagem ou lixamento), pois ocorre o aquecimento da superfície trabalhada assim como do particulado produzido. Portanto, para determinar o grau de periculosidade do particulado sólido do aglomerado quanto à concentração de formaldeído $\left(\mathrm{C}_{\mathrm{f}}\right)$, deve-se considerar o tempo de exposição; a variabilidade intraindividual, pois o 
trabalhador pode ser mais ou menos suscetível à substância; a eficiência do sistema de exaustão existente sobre o maquinário; e a distância do trabalhador ao maquinário.

Tabela 2. Síntese dos valores da concentração de sílica $\left(\mathrm{C}_{\mathrm{s}}\right)$ e concentração de formaldeído $\left(\mathrm{C}_{\mathrm{f}}\right)$ obtidos no ambiente de trabalho analisado na presente pesquisa.

Table 2. Value synthesis of silica concentration $\left(\mathrm{C}_{\mathrm{s}}\right)$ and formaldehyde concentration $\left(\mathrm{C}_{\mathrm{f}}\right)$ obtained in work environment under analysis in this research.

\begin{tabular}{llll}
\hline Especificação das informações levantadas & $\begin{array}{c}\text { Concentração } \\
\text { média }\left(\mathbf{m g} / \mathbf{m}^{\mathbf{3}}\right)\end{array}$ & $\begin{array}{c}\text { Desvio padrão / } \\
\mathbf{d p}\left(\mathbf{m g} / \mathbf{m}^{\mathbf{3}}\right)\end{array}$ & $\begin{array}{c}\text { Parâmetro de } \\
\text { comparação }\left(\mathbf{m g} / \mathbf{m}^{\mathbf{3}}\right)\end{array}$ \\
\hline $\begin{array}{l}\text { Concentração de sílica cristalina na amostra }=\mathrm{C}_{\mathrm{s}} \\
\text { (poeira total - PT). }\end{array}$ & $\mathrm{C}_{\mathrm{s}}=0,0592$ & 0,0215 & $\mathrm{LT}_{(\mathrm{ACGIH})}=0,022$ \\
$\begin{array}{l}\text { Concentração de sílica cristalina na amostra }=\mathrm{C}_{\mathrm{s}} \\
\text { (poeira respirável - PR). }\end{array}$ & $\mathrm{C}_{\mathrm{s}}=0,0043$ & $* * *$ & $\mathrm{LT}_{(\mathrm{ACGIH})}=0,022$ \\
$\begin{array}{l}\text { Concentração de formaldeído na amostra }=\mathrm{C}_{\mathrm{f}} \\
\text { (poeira total - PT). }\end{array}$ & $\mathrm{C}_{\mathrm{f}}=0,5904$ & 0,2021 & $\mathrm{LT}_{(\mathrm{ACGIH})}=0,369$ \\
\hline
\end{tabular}

$\mathrm{C}_{\mathrm{s}}$ : concentração de partículas de sílica cristalina; $\mathrm{C}_{\mathrm{f}}$ concentração de partículas de formaldeído; $\mathrm{LT}_{(\mathrm{ACGIH})}$ : limite de tolerância da ACGIH. Segundo a ACGIH, se $\mathrm{C}_{\mathrm{s}}>\mathrm{LT}_{\mathrm{ACGIH}}$, existe risco ocupacional. $\mathrm{C}_{\mathrm{f}}>\mathrm{LT}_{\mathrm{ACGIH}}$ : material caracterizado como substância A2 suspeito de desenvolver câncer em seres humanos. ***: concentração definida a partir de uma amostra, portanto sem variação estatística.

Vale salientar que ocorreu, durante os dias de coleta das amostras, variação da temperatura e da umidade relativa do ar, com tendência de redução nos valores obtidos para a massa corrigida do particulado coletado sobre o filtro de membrana. Esse tipo de comportamento do aerodispersoide sólido já havia sido comentado na pesquisa feita por Santos (2005), na qual descreve que o local de deposição da partícula depende do seu tamanho e da sua higroscopicidade, sendo que as partículas menores que $1 \mu \mathrm{m}$ possuem baixo índice de sedimentação pela gravidade. Portanto a redução no valor da massa corrigida das partículas pode ser decorrente da coleta do aerodispersoide pelo sistema de exaustão situado sobre o maquinário. Por essa razão, é recomendado para as empresas do setor de base florestal que utilizam no seu processamento painéis de madeira aglomerada que seja feita uma checagem na regulagem do sistema de exaustão nos dias quentes com baixa umidade do ar e nos dias frios com alta umidade relativa do ar, buscando melhorar a eficiência da coleta desse tipo de aerodispersoide no ambiente de trabalho.

\section{CONSIDERAÇÕES FINAIS}

De acordo com os dados apurados, existe risco ocupacional para o trabalhador exposto por longo período de tempo ao particulado sólido do aglomerado. É importante ressaltar que as amostras do particulado sólido do aglomerado utilizado na presente pesquisa foram coletadas a partir de um estudo de caso, em uma indústria de painéis de madeira aglomerada, visando minimizar algumas varáveis pertinentes ao processamento do material, mas o resultado obtido é aplicável aos demais setores de transformação da indústria de base florestal, mais especificamente a indústria moveleira, responsável pelo consumo de mais de $90 \%$ dos painéis de madeira aglomerada produzidos no Brasil.

A partir da homologação desses resultados, alguns cuidados devem ser tomados pelas empresas do setor de base florestal, buscando minimizar o risco ocupacional sofrido por seus empregados. Com resultado mais imediato, as empresas podem utilizar barreiras de segurança que minimizam a exposição do trabalhador. Esses sistemas de controle podem ser efetuados de duas maneiras: na fonte de propagação do aerodispersoide e/ou diretamente no trabalhador que sofre a exposição.

Como medida de controle do particulado sólido do aglomerado no ambiente de trabalho, é indicada a utilização de um sistema de exaustão eficiente sobre o maquinário, que irá retirar do ambiente de trabalho a poeira no instante do seu processamento. Como ação combinada, deve ser instalado um sistema de exaustão geral na empresa, buscando eliminar a poeira remanescente.

Por outro lado, para controlar a exposição direta do trabalhador, é indicada a utilização de equipamentos de proteção individual (EPIs), que devem ser plenamente adaptáveis aos trabalhadores, para não atrapalhar a realização das tarefas. Em se tratando do particulado sólido do aglomerado, deve-se dar atenção à proteção das mucosas e vias aéreas superiores. Para tanto, é indicado para os trabalhadores 
o uso de óculos e máscaras ou respiradores. Os óculos devem possuir lentes inteiriças, que assegurem a completa proteção do globo ocular, mas com amplo campo de visão e resistentes a impactos. A armação deve ser de material flexível, hipoalérgico, com sistema de ventilação lateral e elástico para retenção à cabeça. As máscaras também devem ser de material hipoalérgico e flexível, ajustável à face do trabalhador. Deve possuir elástico para retenção na cabeça e permitir a troca periódica do sistema de filtros, visando fornecer ao indivíduo um ar livre de partículas de poeira sólida, inclusive as pertencentes à fração respirável.

\section{REFERÊNCIAS}

AMERICAN CONFERENCE OF GOVERNMENTAL INDUSTRIAL HYGIENISTS (ACGIH). Documentation of the Threshold Limit Values and Biological Exposure Indices. Cincinnati, 1991. Disponível em: <http://www.acgih.org>. Acesso em: 5/10/2005.

Threshold Limit Values. Cincinnati, 1998 Disponível em: 〈http://www.acgih.org〉. Acesso em: 5 5/10/2005.

. Threshold Limit Values and Biological Exposure Indices. Cincinnati, 2006. 236 p.

AMERICAN SOCIETY FOR TESTING AND MATERIALS (ASTM). ASTM D4690-99. Standard Specification for Urea-Formaldehyde Resin Adhesives. USA, 2005. Disponível em: <http://www.astm.org>. Acesso em: 13/10/2006.

ASTM E112-96. Standard Test Methods for Determining Average Grain Size, (Reapproved 2004). USA, 2004. Disponível em: <http://www.astm.org>. Acesso em: 13/10/2006.

ASTM E1382-97. Standard Test Methods for Determining Average Grain Size Using Semiautomatic and Automatic Image Analysis, (Reapproved 2004). USA, 2004. Disponível em: <http://www.astm.org>. Acesso em: 13/10/2006.

BRITISH STANDARDS. BS EN 312:2003. Particleboards. Specifications Part 4: Requirements for loadbearing boards for use in dry conditions. London, 2003. German version EN 312-4:2003.

BURGER, L. M.; RICHTER, H. G. Anatomia da madeira. São Paulo: Nobel, 1991.

FUNDAÇÃO JORGE DUPRAT FIGUEIREDO DE SEGURANÇA E MEDICINA DO TRABALHO (FUNDACENTRO). NHO 03 - Análise Gravimétrica de aerodispersóides sólidos coletados sobre filtros de membrana. Norma de Higiene Ocupacional. Brasília, DF, 2001.

IWAKIRI, S. Painéis de madeira reconstituída. Curitiba: FUPEF, 2005.

NATIONAL INSTITUTE FOR OCCUPATIONAL SAFETY HEALTH (NIOSH). Documentation of the threshold limit values. Atlanta, 1992. Disponível em: 〈http://www.cdc.gov/niosh>. Acesso em: $5 / 10 / 2005$.

Method 2016. Formaldehyde. Atlanta, 2003. Disponível em: 〈http://www.cdc.gov/niosh〉. Acesso em: 5/10/2005.

Method 7602. Sílica, Crystalline by.IR (K BR pellet). Atlanta, 2003. Disponível em: <http://www.cdc.gov/niosh>. Acesso em: 5/10/2005.

Method 500. Particulates not Otherwise Regulated, Total, Issue 2. Atlanta, 1994.. Disponível em: <http://www.cdc.gov/niosh>. Acesso em: 5/10/2005.

Method 600. Particulates not Otherwise Regulated, Respirable, Issue 3. Atlanta, 1998. Disponível em: <http://www.cdc.gov/niosh>. Acesso em: 5/10/2005.

OCCUPATIONAL SAFETY \& HEALTH ADMINISTRATION (OSHA). Exposicíon a la sílica cristalina. Washington, DC, 2002. Disponível em: <http://www.osha.gov/publications/3179-2002>. Acesso em: 5/10/2005. 
Gravimetric Determination. PV 2121/ Partially Validated 2121. Washington, DC, 2003. Disponível em: <http://www.osha.gov/dts/sltc/methods/toc.html>. Acesso em: 5/10/2005.

Permissible Exposure Limit. Washington, DC, 1994. Disponível em:〈http://www.osha.gov> Acesso em: 5 out 2005.

OCCUPATIONAL SAFETY AND HEALTH REVIEW COMMISSION (OSHC). Disponível em: <http://www.oshrc.gov>. Acesso em5/10/2005.

SANTOS, A. M. A. Exposição ocupacional a poeiras em marmorarias: tamanhos de partículas característicos. Tese (Doutorado em Engenharia Metalúrgica) - Universidade Federal de Minas Gerais. Belo Horizonte, 2005. Disponível em: $<$ http://www.fundacentro.gov.br/dominios

/CTN/teses_conteudo.asp?retorno=120>. Acesso em: 20/08/2007. 